\title{
Special Clinical Characteristics of Struma Ovarii- Two Unique Cases Report
}

\author{
Yi Liang Lee ${ }^{1}$, Yin Shiun Bai ${ }^{2}$ and Chang Sheng Yin ${ }^{2 *}$ \\ ${ }^{1}$ Department of Obstetrics and Gynecology, Cathay General Hospital, Taiwan \\ ${ }^{2}$ Department of Obstetrics and Gynecology, Kang Ning Hospital, Taiwan
}

*Corresponding author: Chang Sheng Yin, Department of Obstetrics and Gynecology, Kang Ning Hospital, no.26, lane 420, sec 5, Cheng Kung Rd, Neihu, Taipei, Taiwan.
Received Date: March 26, 2019

Published Date: March 29, 2019

\begin{abstract}
Introduction: Struma ovarii is a rare type of ovarian cystic teratoma with composed of predominantly thyroid tissue. It occurs between 30-50 years of age, usually in unilateral ovary. Struma ovarii rarely occurs before puberty and is growing slowly. Hereby we reported 2 cases in which one case with combined cystic teratoma in one side and Struma ovarii in contralateral side of ovary, the second case with Struma ovarii occurs at age of 16 then growing tremendously with in only 8 years.
\end{abstract}

Methods: A retrospective study of the data bases of community hospitals in Taipei city during 2014 -2016 were included. Two cases with a histological diagnosis of Struma ovarii who had undergone surgery were identified.

\section{Patients}

Case 1: A 29-year-old female patient presented with recently irregular vaginal bleeding, abdominal distention and low abdominal palpable mass. Her past history revealed right ovarian cystic teratoma underwent laparoscopic removal of tumor at age of 16 . CT scan revealed the contralateral ovarian calcified tumor $(10 \mathrm{~cm} \times 7.5 \mathrm{~cm})$ with heterogenous mass with solid and cystic components. Post-operative finding was left ovarian cyst with smooth surface and gelatinous material content.

Case 2: A 24-year-old single patient presented with amenorrhea and abdominal distention. She had history of right ovarian simple cyst $(4.2 \mathrm{~cm} \times 3.2 \mathrm{~cm})$ at age of 16 . She came after 8 years; the physical examination found the ovarian tumor growing to a tremendous size. CT scan showed a homogenous cystic tumor $25.8 \mathrm{~cm}$ x $25.7 \mathrm{~cm}$ x $16 \mathrm{~cm}$ with a few heterogenous enhanced nodules. Post-operative finding of big left ovarian cystic tumor, glistening surface with gelatinous material, few septations was observed

All two patient's pre-operative diagnosis were dermoid tumors with suspected ovarian malignancy.

Conclusion: Due to the rarity of Struma ovarii its natural history, growth pattern and treatment strategy is not yet cleared. Clinical presentation and symptoms of Struma ovarii are vary, image studies are often suspected of ovarian carcinoma. Preoperative diagnosis is important. The best management plan should be individualized. More cases reports are needed.

Keywords: Cystic teratoma; Fast growing Struma ovarii

\section{Introduction}

The Struma ovarii is rare, a type of mono-dermic teratoma which composed entirely or predominantly of thyroid tissue, constitutes approximately $3 \%$ of all ovarian teratomas, $1 \%$ of all ovarian tumors [1]. Struma ovarii occurs between 30-50 years of age, usually in unilateral ovary, very rarely in combination of contralateral ovarian cystic teratoma. Struma ovarii rarely occurs before puberty and growing slowly. Hereby we reported 2 cases in which one case with combined cystic teratoma in one side and Struma ovarii in contralateral ovary, the second case with Struma ovarii occurs at age of 16 then growing tremendously within 8 years.

\section{Materials and Methods}

Women with a histological diagnosis of Struma ovarii who had undergone surgery were identified from the data bases of 
community hospitals in Taipei city during 2014 -2016 were included. The mini-laparotomy approach for large ovarian cysts suggested by Pelosi MA II, et al [2] and was detailed elsewhere [3]. Two patients with histologically confirmed Struma ovarii whose medical records were retrospectively reviewed and analyzed.

\section{Results}

All surgeries were by mini-laparotomy in case 1 with RSO, and case 2 with ophoro-cystectomy. All two patient's pre-operative diagnosis were dermoid tumors with suspected ovarian malignancy.

\section{Case 1}

A 29-year-old single lady presented with recently irregular vaginal bleeding and abdominal distention and low abdominal palpable mass. On examination, the temperature was $36.8^{\circ} \mathrm{C}$, the blood pressure $134 / 90 \mathrm{mmHg}$, the heart rate 82 beats per minute, the respiration rate breaths 17 per minute. Her thyroid was not enlarged. The weight was $54 \mathrm{~kg}$, the height $168 \mathrm{~cm}$ and the BMI 21 . Her past history revealed right ovarian cystic teratoma underwent laparoscopic removal of tumor at age of 16. Two years before admission, she was incidentally identified a left ovarian tumor which was $10 \mathrm{~cm} \times 7.5 \mathrm{~cm}$ in size at this clinic. During that time, she declined surgery and then follow up at clinic. Before admission, the ultrasound and CT scan revealed a left adnexal calcified tumor with heterogenous mass with solid and cystic components, size $10.7 \mathrm{~cm}$ $x 7.7 \mathrm{~cm}$ without ascites. All the three tumor markers, (CA-125 $31 \mathrm{u} / \mathrm{ml}$, AFP $1.58 \mathrm{ng} / \mathrm{ml}$, CEA $1.1 \mathrm{mg} / \mathrm{ml}$ ) was within normal limits. She underwent mini-laparotomy with left ovarian cystectomy. Intraoperative finding was left ovarian cyst with smooth surface with gelatinous material content.

\section{Case 2}

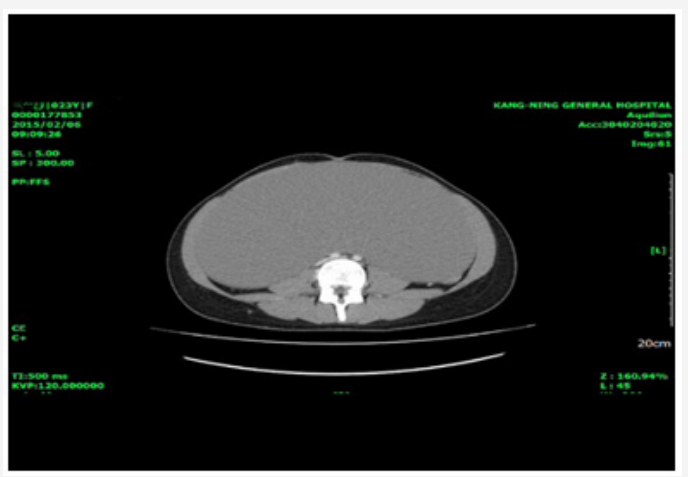

Figure 1: Case 2, huge homogenous cyst, $25.8 \mathrm{~cm} \times 25.7 \mathrm{~cm}$.

A 24-year-old single lady presented with amenorrhea for 4 months and abdominal distention. Eight years before presentation, at age of 16 , she called to our clinic for amenorrhea and was incidentally found to have a right ovarian simple cyst $(4.2 \mathrm{~cm}$ $\mathrm{x} 3.2 \mathrm{~cm}$ ). Her grandmother had undergone total abdominal hysterectomy and bilateral salpingo-oophorectomy 25 years ago due to clear cell carcinoma of ovary. On examination, the temperature was $36 \circ \mathrm{C}$, the blood pressure $115 / 86 \mathrm{mmHg}$, the heart rate 80 beats per minute, the respiration rate 18 breaths per minute. The weight was $64 \mathrm{~kg}$, the height $168 \mathrm{~cm}$ and the BMI 22.6 . Blood levels of TSH (2.31 u IU/ml), and ovarian tumor markers was normal (CA-125 31.9u/ml, AFP 2.59ng/ml, CEA $1.2 \mathrm{mg} / \mathrm{ml}$, Beta-
HCG $0.1 \mathrm{~m} \mathrm{IU} / \mathrm{ml}$ ). The abdominal examination revealed a big mass to low level of xiphoid process. Ultrasonography of the abdomen and CT scan showed a giant homogenous cystic tumor $25.8 \mathrm{~cm}$ x $25.7 \mathrm{~cm} \times 16 \mathrm{~cm}$, a few heterogenous enhanced nodules. She underwent mini-laparotomy with left salpingo-oophorectomy was done (Figures 1\&2). Operative finding: big left ovarian cystic tumor, glistening surface with gelatinous material, few septations. Uterus and right-side tube and ovary were normal. Following removal, the collapsed cyst was refilled with $6750 \mathrm{~mL}$ of normal saline to demonstrate its actual size.

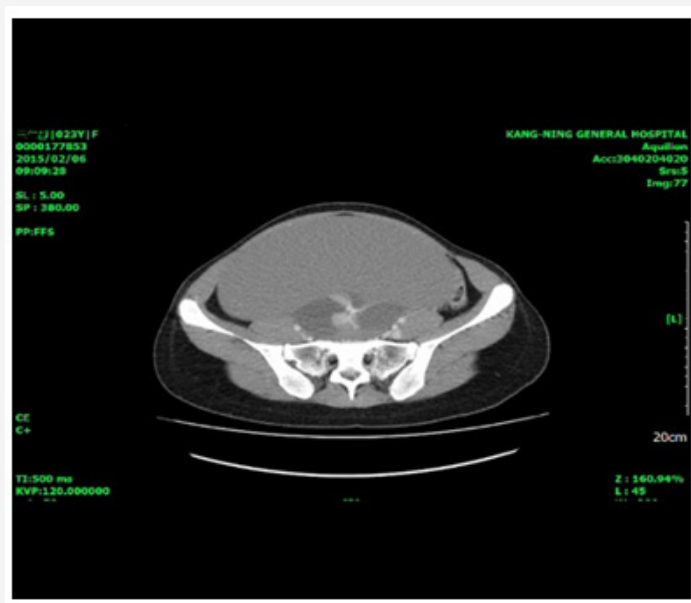

Figure 2: Case 2, few heterogenous enhanced area and solid components in low part.

There was no patient with tachycardia or other manifestations of hyperthyroid function, and no thyroid tumors was found by ultrasonography.

The age of the two patients were 29 and 24 respectively, both were of fertile age. Case 1 was abnormal uterine bleeding, case 2 presented with amenorrhea, both without thyrotoxicosis. No patient had ascites. Case 2 had a family history of ovarian cancer, neither had been operated on for breast cancer or other diseases.

\section{Discussion}

In this report case 1 was a rare occurrence, cystic teratoma in right ovarian cystic teratoma that was removed at age of 16 , while left ovarian Struma ovarii removed at age of 29. Qiao, et al [3] reported a similar case, the tumor in the right ovary consisted Struma ovarii, while in the left ovary was a cystic teratoma, both tumors removed at same time [3]. Deguchy, et al [4] reported 2 cases of rapid growing cystic teratoma and concluded that: it has been shown that classic features of a cystic teratoma slow growth at an average rate of $1.8 \mathrm{~mm}$ each year in premenopausal patients, but more rapid growth should not exclude the diagnosis of a cystic teratoma [5]. The Case 2 was interesting: at age of 16 her right ovarian cyst was found incidentally just $4.2 \mathrm{~cm} \times 3.2 \mathrm{~cm}$ then after 8 years later the tumor growing to tremendous size of $25.8 \mathrm{~cm} \times 25.7 \mathrm{~cm}$, a growth of almost $25 \mathrm{~cm}$ in an 8 -year timeframe, or $30 \mathrm{~mm} /$ year. No previous report concerning the growing pattern of Struma ovarii was noted. Hyperthyroidism in the approximately $8 \%$ patients, but signs or symptoms of thyrotoxicosis is rare. Since strumal ovarii tissue is usually not functionally active, the associated with thyrotoxicosis can be due to autoimmune stimulation of the normal thyroid gland. 
The natural history and optimal treatment strategy for Struma ovarii remains unclear due to its rarity.

The clinical presentation and symptoms of Struma ovarii are similar to those of other ovarian tumors and images are often suspected of ovarian carcinoma. Yoo, et al. [6] in 2008 reported that the most common initial symptoms are abdominal pain and distension, a palpable mass. and or vaginal bleeding [5]. Our Case 1 presented with irregular vaginal bleeding, and abdominal distention, while Case 2 was amenorrhea for 4 months and big palpable abdominal mass.

Preoperative diagnosis is important to avoid ovarian cancer type surgery. Diagnosis must be made by histopathological examination as intraoperative naked eyes diagnosis is not reliable. It is difficult to distinguish between Struma ovarii and dermoid cysts on the basis of their sonographic appearance. Yoo, et al. [6] found that ultrasonography was able to diagnose Struma ovarii in only 4/34 (11.8\%) patients [5]. Mustafa reported a case of Struma ovarii was diagnosed by preoperative scintigraphy with radioactive iodine [131I] which showed active thyroid tissue in pelvis [6]. Zalel, et al. [7] demonstrated the capsule of dermoid cysts are usually devoid of blood flow, when low resistance Doppler flow signals, detected from the center of the echoic lesion (Struma pearl), may be more common in Struma ovarii [8].

The presence of thyroid tissue in Struma ovarii may demonstrate the appearance of high-density viscous colloid cysts containing thyroglobulin and thyroid hormones in the tumor resulting of low signal intensity are seen within the cyst on radiographic scan. Zhang, et al. [9] summarized computed tomography diagnostic clues included:

1. Mostly cystic-solid type, solid mass is rare;

2. Uniform density;

3. High-density cysts might be a characteristic feature;

4. Does not demonstrate lipid material;

5. Smooth cyst wall, calcified septum;

6. Marked enhancement of the thick septations/solid components/thyroid tissue with contrast agent;

7. Always show small amount of ascites.

While on pelvic magnetic resonance imaging included:

1. Low signal in T1W1 and high in T2W1 of cyst fluid;

2. Variety of signal intensities on T1-/T2-weighted images of thyroid tissue/thickened septs /cyst walls;

3. Very low intensity on T2-weighted MR and no enhancement on contrast-enhanced MR of cystic areas [8].

Malignant Struma ovarii is rare, accounts for $0.1-0.3 \%$ of mature teratomas cases. While metastasis from malignant Struma ovarii has only been reported in 5-6\% of malignant Struma ovarii cases [9]. A study of 88 patients with malignant Struma ovarii, the adverse clinical course was noted as:

1. Adhesions formation (graded 2 to $4+$ ),
2. Peritoneal fluid ( $>$ or $=1 \mathrm{~L}$ ), 3. ovarian serosal rent [9].

In a study of 68 patients with malignant Struma ovarii, (MSO) Goffredo et al found excellent disease-specific survival rates for the condition no matter what surgical treatment was used [10]. But MSO patients had a high risk for developing aggressive thyroid cancers, routine thyroid imaging in these patients were needed. Due to the rarity of malignant Struma ovarii [11], the development of new biomarkers is required.

The limitations of our study include Struma ovarii is a rare condition, hard to collect a large number of cases over a long period of time, and from different centers in order to obtain a reasonably large sample. Clinical manifestations and treatment options depend only on cases or case series. Currently there is no consensus on which the best management and each case is must be managed individually.

In summary, in this cases report, we have described special clinical features of Struma ovarii in a group of 2 reproductive aged ladies. More cases reports are needed.

\section{Acknowledgement}

The authors would like to thank Miss Feng-Wei Bai, Pei-Ying Yang RN, Miss LI-Chuan Yao and for their hard work and support throughout this study.

\section{Conflict of Interest}

No conflict of interest.

\section{References}

1. Talerman A (2001) Germ cell tumors of the ovary. In Blaustein's Pathology of the Female Genital Tract ( $3^{\text {rd }}$ edn), Kurman RJ (edts), Springer Verlag: New York, USA, pp. 967-1033.

2. Pelosi MA II, Pelosi MA III (2004) A novel minilaparotomy approach for large ovarian cysts. OBG Manag 16(2): 17-30

3. Qiao PF, Gao Y, Niu GM (2015) Struma ovarii accompanied by mature cystic teratoma of the other ovary: A case report and literature review. Oncol Lett 9(5): 2053-2055.

4. Deguchy Q Jr, Fananapazir G, Corwin M, Lamba R, Gerscovich E, et al. (2016) Benign Rapidly Growing Ovarian Dermoid Cysts: A Case Series. Journal of Diagnostic Medical Sonography 33(1): 71-74.

5. Caspi B, Appelman Z, Rabinerson D, Zalel Y, Tulandi T, et al. (1997) The growth pattern of ovarian dermoid cysts: a prospective study in premenopausal and postmenopausal women. Fertil Steril 68(3): 501505 .

6. Yoo SC, Chang KH, Lyu MO, Chang SJ, Ryu HS, et al. (2008) Clinical characteristics of struma ovarii. J Gynecol Oncol 19(2): 135-138.

7. Zalel Y, Seidman DS, Oren M, Achiron R, Gotlieb W (2000) Sonographic and clinical characteristics of struma ovarii. J Ultrasound Med 19(12): 857-861.

8. Matsuki M, Kaji Y, Matsuo M, Kobashi Y (2000) Struma ovarii: MRI findings. Br J Radiol 73(865): 87-90.

9. Zhang T, Chen PP, Gao Y (2018) Review Article. Struma ovarii: a mini review. Int J Clin Exp Med 11(10): 10364-10371.

10. Robboy SJ, Shaco-Levy R, Peng RY, Snyder MJ, Donahue J, et al (2009) Malignant struma ovarii: an analysis of 88 cases, including 27 with extraovarian spread. Int J Gynecol Pathol 28(5): 405-422.

11. Goffredo P, Sawka AM, Pura J, Adam MA, Roman SA, et al. (2015) Malignant struma ovarii: a population-level analysis of a large series of 68 patients. Thyroid 25(2): 211-215. 